\title{
AssistLibras: Um Assistente Gráfico para Construção de Sinais 3D da LIBRAS
}

\author{
Denys Fellipe Souza Rocha
}

\author{
Orientadores: Ig Ibert Bittencourt, Patrick Brito
}

Instituto de Computação - Universidade Federal de Alagoas (UFAL)

\author{
Campus A. C. Simões - Av. Lourival Melo Mota, s/n, Tabuleiro dos Martins - Maceió - \\ AL, CEP:57072-900 \\ contato.denysrocha@gmail.com, \{ig.ibert, Patrick\}@ic.ufal.br
}

\begin{abstract}
Resumo. A sociedade contemporânea se compreende em padrões de massa, onde a melhor decisão é aquela que atende a maior parte das pessoas, deixando de fora quem está nas margens. O termo deficiência está associado a limitações, visto de forma pejorativa como ausência de habilidades/competências. O entendimento do conceito de Acessibilidade vem a tornar claro o lugar de cada membro da nossa sociedade, onde todos possuem necessidades diferentes, e o ambiente deve propor a inclusão. No caso do surdo não é diferente, temos a ausência de sua inclusão, que se daria por meio da disseminação de sua língua nativa, a Língua de Sinais. Uma forma de promover essa inclusão seria através da construção de tecnologias assistivas adequadas a sua realidade. A proposta deste TCC é a construção e utilização de uma ferramenta que irá facilitar a confecção de sinais $3 D$ da LIBRAS (Lingua Brasileira de Sinais), o AssistLibras, já que não existe tal ferramental no mercado ou academia. Os sinais nele produzidos serão inseridos no tradutor FALIBRAS, tendo como foco a situação citada anteriormente. Com a utilização desta ferramenta, dar-se-á condições para um usuário leigo, manusear o personagem proposto de forma fácil e intuitiva, gerando sinais legíveis ao surdo. E como validação do trabalho foi realizado um estudo de caso com um surdo, onde foram feitos alguns testes com um(a) professora(a) da LIBRAS, um usuário comum desconhecedor do processo de animação e da língua em questão, onde os mesmos criaram alguns sinais, e o surdo como avaliador da ferramenta.
\end{abstract}

Abstract. Contemporary society is understood in mass standards, where the best decision is one which conforms to most people, leaving out those who are on the margins. The term disability is associated to the limitations, pejoratively as lack of skills / competencies. Understanding the concept of accessibility is to make clear the place of every member of our society, where everyone has different needs, and the environment should propose inclusion. In the case of the deaf is no different, we have the absence of its inclusion, it would get through the dissemination of their native language, sign language. One way to promote such inclusion would be through the construction of appropriate assistive technologies to their reality. The purpose of this final paper is to present the making, and use, of a tool intended to facilitate the construction of $3 D$ representations for LIBRAS (Lingua Brasileira de Sinais - 
Brazilian Sign Language), named AssistLibras, since there is no such tooling on the market or academia. The signals produced in AssistLibras are to be used by in FALIBRAS translator. The use of the tool allows users with no or very little experience, handling the proposed character in an easy and intuitive way, generating readable signs for deaf. Several signs were produced using the characters and to validate the work, we have performed a case study with a deaf where some tests were performed with (a) teacher (a) of LBS, a common user unaware of sign language and the animation process, and a deaf person as the tool evaluator.

\section{Introdução}

O grande problema do surdo é que apesar de toda a evolução tecnológica, o mesmo continua sendo excluído da sociedade, que por não saber o que fazer para promover a comunicação com ele, simplesmente o ignora negando seus direitos de cidadania mais necessários, como é o caso da educação. Torna-se evidente o reconhecimento do real sentido da palavra "Acessibilidade", não apenas ter ciência de seu significado, mas proporcionar sua prática através das atuais TICs (Tecnologias de Informação e Comunicação), como por exemplo, não há hoje uma ferramenta consolidada que facilite a construção de sinais da LIBRAS, matéria prima da comunicação do surdo brasileiro.

Segundo o último censo do IBGE (2010) "a deficiência auditiva acometia 9,7 milhões de pessoas $(5,1 \%)$, sendo que a deficiência auditiva severa (pessoas com grande dificuldade ou incapazes de ouvir) foi declarada por 2,1 milhões de pessoas, das quais 344,2 mil eram surdas (0,2\%)." Mundialmente são 70 milhões de surdos, 30\% dos surdos brasileiros não sabem ler o Português, os restantes $70 \%$ tem um conhecimento restrito da língua, e especialmente no Estado do Piauí 95\% dos surdos são analfabetos. Com a lei $n^{\circ} 10.436$, chamada de Lei de LIBRAS, a prática e o acesso à língua de sinais se torna obrigatória nas instituições onde houver algum surdo, cujo artigo $2^{\circ}$ diz: "Deve ser garantido, por parte do poder público em geral e empresas concessionárias de serviços públicos, formas institucionalizadas de apoiar o uso e difusão da Língua Brasileira de Sinais - LIBRAS como meio de comunicação objetiva e de utilização corrente das comunidades surdas do Brasil." (BRASIL, 2002)

O sistema escolar brasileiro não foi preparado para receber pessoas que possuam alguma necessidade especial, assim a educação fica restrita a um grupo padrão, quem estiver fora desse padrão, é excluso do direito de aprender. Como retrata Frias e Menezes (2008): “Assim, a escola historicamente se caracterizou pela visão da educação que delimita a escolarização como privilégio de alguns grupos, legitimando um processo de exclusão através de suas políticas e práticas educacionais, que reproduzem a ordem social."

Diante do exposto pode-se levantar o seguinte questionamento: De que forma a tecnologia pode promover acessibilidade do surdo facilitando a sua comunicação com o seu meio social?

Através da construção de uma ferramenta computacional, com uma tecnologia assistiva adequada a sua realidade, será possível promover a comunicação do surdo com seu meio social com a forma final de um tradutor de LIBRAS, para que o surdo possa ter acessibilidade. 
O objetivo deste trabalho é promover um meio de inclusão digital e social do surdo brasileiro, através de uma ferramenta que produza os sinais da LIBRAS com qualidade e legibilidade, onde estes serão inseridos e utilizados em um tradutor.

A pesquisa se dá de forma teórica, experimental e aplicada, pois como primeira fase da proposta, temos a criação da ferramenta, levando em consideração os parâmetros da língua de sinais escolhida, padrão gráfico adotado com o personagem, e avaliação de usabilidade da ferramenta. Já na segunda fase, temos os testes realizados com: um professor de língua de sinais, produzindo os mesmos; um usuário leigo em computação gráfica e LIBRAS, confeccionando os sinais com o auxílio de um guia; e um surdo que avalia o produto final, ou seja, os sinais já dentro do tradutor.

\section{AssistLibras: Uma ferramenta computacional para geração de sinais da}

\section{LIBRAS em 3D}

O AssistLibras é um assistente de construção de sinais da LIBRAS. O mesmo foi projetado para que qualquer leigo em computação gráfica possa manuseá-lo de forma bastante fácil e intuitiva.

O assistente é uma interface projetada no software 3D Studio Max, na forma de um arquivo com configurações fixas e um layout personalizado, próprio para a criação dos sinais. Nele é apresentado um personagem padrão, uma menina chamada "Sophya", uma personagem estilo cartoon. Isso faz parte de uma estratégia de mercado, pois personagens humanoides nunca ficam fiéis e parecem um tanto robóticos, causando uma antipatia ao usuário. Já quando se trata de um personagem cartoon, com características lúdicas e comuns a desenhos animados, fazendo com que o usuário se identifique logo em seguida.

A personagem é vista de três ângulos diferentes, para que na hora da confecção do sinal, o usuário tenha uma boa visualização espacial do movimento, como visto na Figura 1. Possui uma estrutura esquelética pré-definida pelo 3Ds Max, com todas as restrições de movimento de um humano.

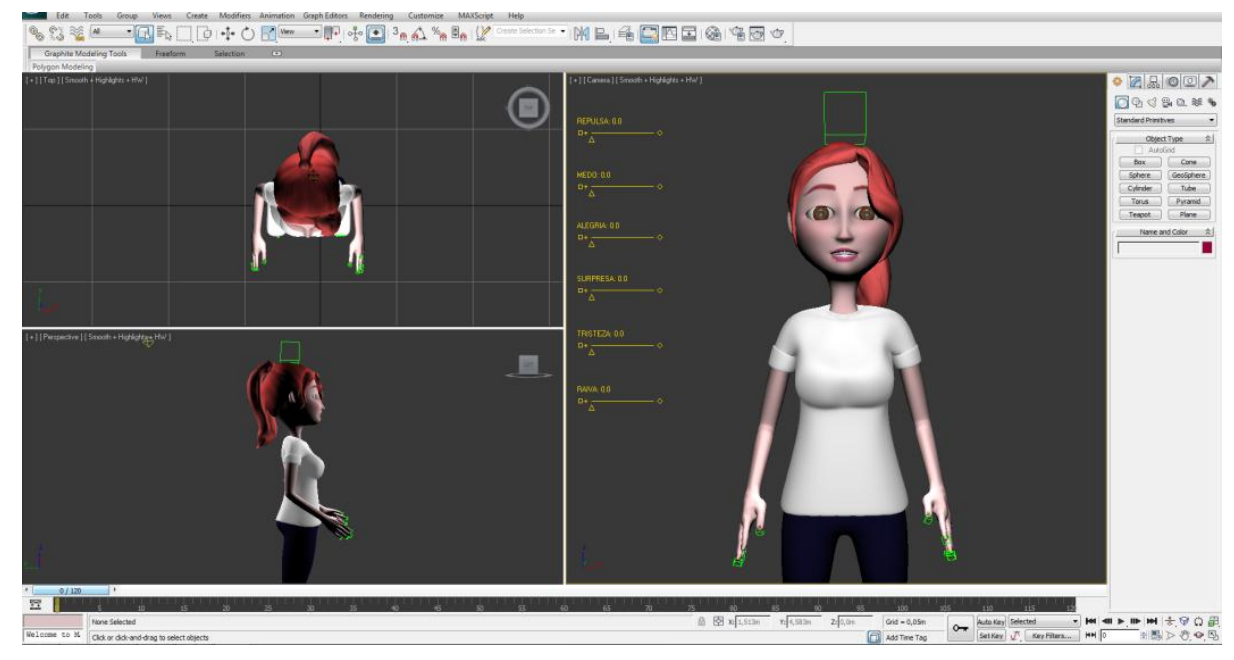

Figura 1: Inteface inicial do AssistLibras 
No AssistLibras, o usuário deve ter apenas uma breve noção de animação e Time Line (Linha de Tempo), pois é nisto que consiste a criação de cada sinal. Ao final, o propósito de sua utilização é criar uma biblioteca robusta, que possa ser utilizada em algum tradutor.

Apesar do assistente ter sido projetado para que tudo se torne mais fácil, a qualidade do sinal irá depender muito do manuseio do animador, pois neste campo, o usuário precisa exercitar sua habilidade para que os movimentos produzidos fiquem realmente fiéis aos sinais na vida real, ou seja, fiquem naturais.

\subsection{Especificação de Requisitos e Visão Geral da Ferramenta}

O AssistLibras pode ser considerado um ambiente de geração de atores virtuais articulados, pois nele temos a modelagem de um personagem, que por sua vez pode ser trocado, geração de movimentos com base em uma estrutura composta por restrições, e o resultado final que é uma cena renderizada. Como retrata Schneider e Nedel:

"um sistema capaz de gerar um ator virtual articulado deve considerar quatro
etapas: (i) a modelagem do objeto (determinação das articulações e dos graus
de liberdade do objeto - uniaxiais, biaxiais ou poliaxiais); (ii) a geração das
ações (definição dos gestos necessários para exprimir ideias ou sentimentos);
(iii) o carregamento da cena (renderização da cena e manipulação dos dados
dos gestos); e (iv) a geração do movimento." (2006)

Não é necessário ter conhecimento de programação, pois é tudo feito ao arrastar do mouse. E como mencionado acima, não ficamos presos a "Sophya", pois podemos modelar outros personagens, e reusar toda a configuração do personagem atual, restando como trabalho somente realizar o "rigging", que nada mais é que associar a malha ao esqueleto. Esse fator abre possibilidades da criação de personagens que se adequem a todas as realidades, ou seja, personagens de diferentes raças e culturas.

\subsection{A Interface de geração de animações}

O nosso assistente gráfico foi criado para que uma língua fosse inserida no mesmo. Ou seja, temos presente no assistente uma interface projetada com os cinco parâmetros da Língua Brasileira de Sinais.

Para criar um sinal no AssistLibras temos uma série de passos bastante simples, onde será mostrada essa interface e sua relação com os parâmetros da língua. (Ver sequência de passos na versão completa do texto).

Segundo Jaques e Viccari (2005a) entende-se emoção como um grupo de estados afetivos genéricos, onde pode-se encontrar também o humor. Diferente do humor, a emoção se resume a momentos breves, que são voluntários ou involuntários.

As expressões foram construídas seguindo um padrão de emoções, onde Ekman (1999) elenca seis emoções básicas que possuem a mesma manifestação facial independente da cultura dos indivíduos que as expressam, como mostrado na Figura 2. 


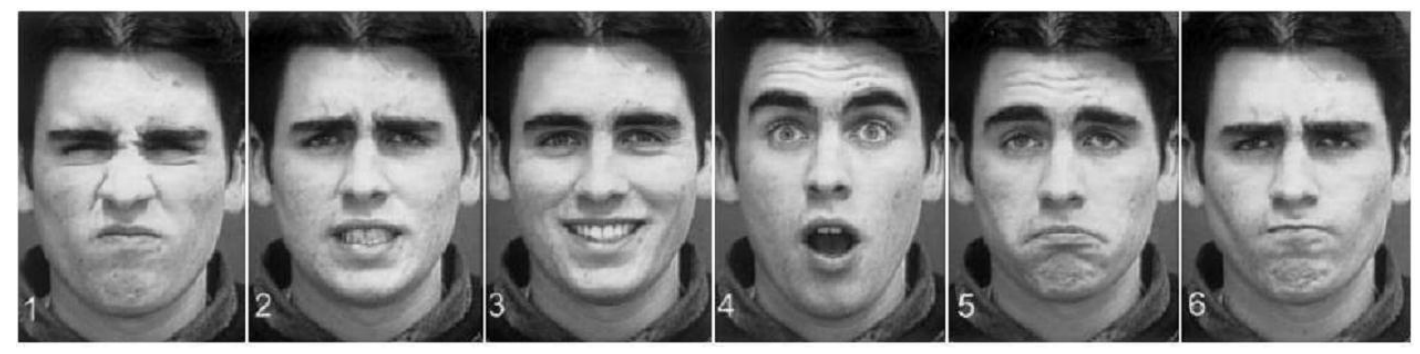

Figura 2: Seis expressões faciais emocionais básicas: (1) repulsa, (2) medo, (3) alegria, (4) surpresa, (5) tristeza e (6) raiva. Schmidt e Cohn (2001)

Seguindo esse padrão, as expressões da Sophya foram construídas de forma a serem o mais fieis às apresentadas por Ekman, garantindo o entendimento correto das emoções por parte do surdo, como observado na Figura 3.

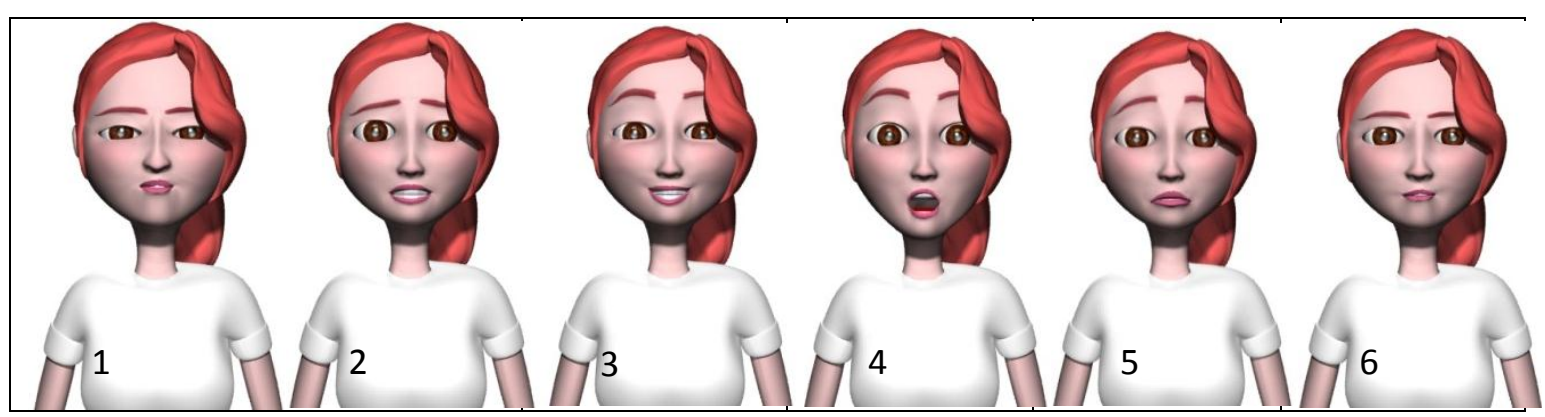

Figura 3: Expressões da personagem Sophya segundo o padrão de Ekman (1999)

Pode-se perceber claramente a importância de uma boa interface projetada para usuários leigos em animação gráfica, e até mesmo na língua de sinais, pois o usuário pode ser um intérprete da língua, com também um usuário "curioso" que através do website do INES (Instituto Nacional da Educação do Surdo), pode observar o sinal e reproduzi-lo com fidelidade.

O AssistLibras não é somente uma interface que produz animação, mas uma interface que possui uma língua e suas características gramaticais e semânticas embutidas, e reproduz essa língua com fidelidade, sem que seja necessário qualquer conhecimento técnico apurado.

\section{Avaliação}

A seguir, será mostrado os resultados dos experimentos realizados com a professora de LIBRAS, o usuário leigo e o surdo, no AssistLibras, através de gráficos detalhados e argumentados.

\subsection{Com professora de LIBRAS}

No gráfico a seguir será mostrada uma comparação entre dois fatores: Tempo e Qualidade de cada animação gerada. O conceito de pontuação admitido para demonstrar o fator "qualidade" foi: 2 (ruim); 4 (regular); 6 (bom); 8 (ótimo); 10 (excelente). Tais resultados foram adquiridos por análise quantitativa e qualitativa do autor da ferramenta juntamente ao usuário em questão. 
Depois de um breve treinamento com a professora de LIBRAS Sumaia Maria Silva, a mesma foi capaz de gerar quatro sinais, onde nos gráficos a seguir percebemos a progressão da qualidade dos sinais gerados em relação ao tempo gasto.

\section{Gráfico 1: Geração de sinais pela professora de LIBRAS}

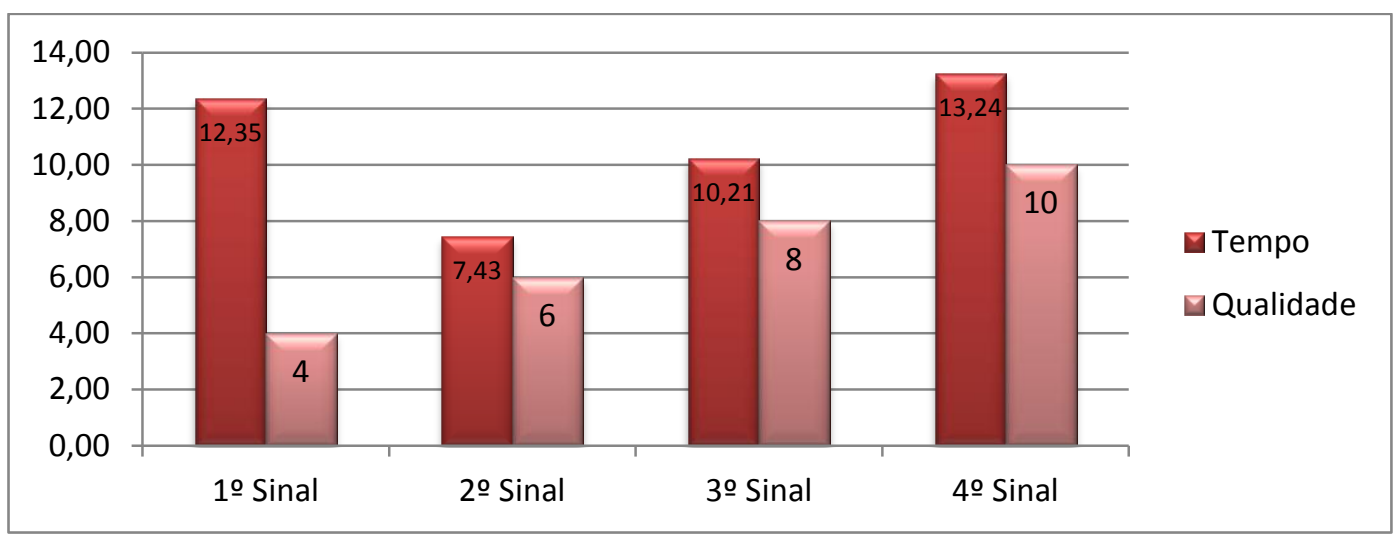

Na geração do primeiro sinal, a palavra "falar", a professora Sumaia gastou 12 minutos e 35 segundos, enquanto que a qualidade do sinal foi de apenas 4 pontos, sendo classificado como "regular", ou seja, a professora ainda estava se familiarizando com a ferramenta.

Já no segundo sinal, a palavra "gostar", percebemos que a mesma ganhou mais habilidade, pois diminuiu o tempo gasto para 7 minutos e 43 segundos, e a qualidade subiu para a nota 6 , dito como "bom".

O terceiro sinal criado foi a palavra "querer", onde a professora se prendeu mais a qualidade do sinal, deixando o tempo maior, com 10 minutos e 21 segundos, sendo o conceito de qualidade 8 , considerado como "ótimo".

O quarto e último sinal gerado foi o próprio nome da professora soletrado, ou seja, "Sumaia". Neste a professora se prendeu a qualidade e não se importou com o tempo gasto na animação, ou seja, o tempo foi de 13 minutos e 24 segundos, enquanto que a qualidade do sinal foi para 10, o conceito máximo: "excelente".

Podemos perceber através desse primeiro experimento, que com a prática de usabilidade da ferramenta, as habilidades do usuário tendem a aumentar de forma linear, e o tempo gasto com cada animação é relativo ao cuidado do usuário em seguir com fidelidade o sinal a ser criado. Também podemos observar que pelo fato de a professora já ser conhecedora da língua, o tempo foi bastante otimizado, restando como limitações apenas a familiaridade com o conceito e práticas de animação gráfica.

\subsection{Com usuário leigo}

Neste experimento, foram produzidos quatro sinais dentro do AssistLibras, onde o usuário Jorge Caetano dos Santos Neto utilizou o site do INES (Instituto Nacional de Educação do Surdo) como guia para a confecção dos mesmos. 
Gráfico 2: Geração de sinais por usuário comum

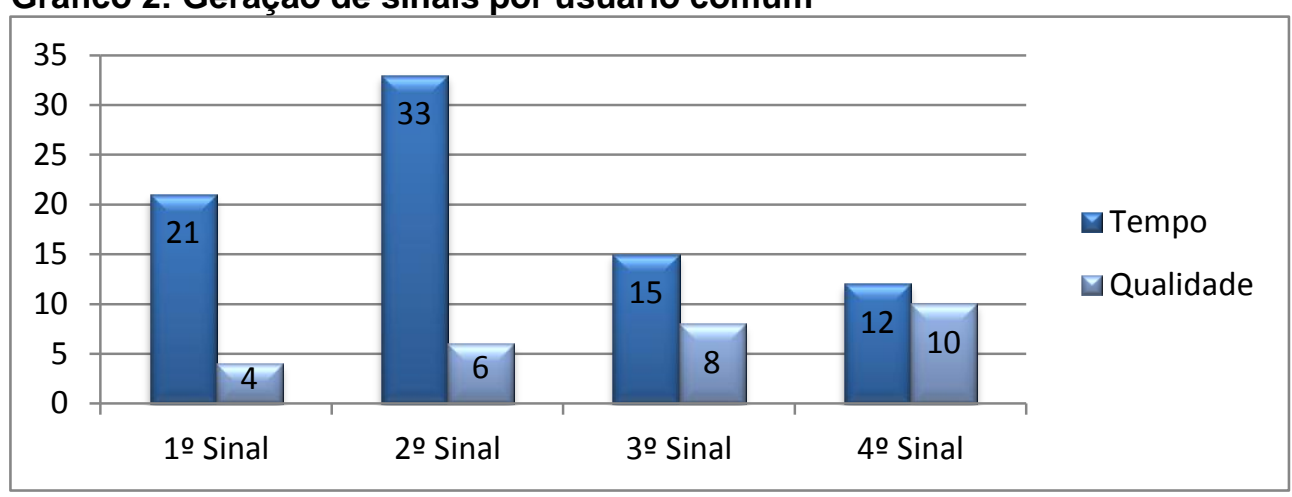

O primeiro sinal produzido foi a palavra "correr". Como primeiro teste, o usuário demonstrou dificuldade com a ferramenta, por não conhecer estar habituado a um software 3D, e ainda estar aprendendo a manusear a interface, gastou 21 minutos e a qualidade foi regular, ou seja, o valor 4 mostrado no gráfico.

No segundo sinal, o usuário demorou mais tempo, 33 minutos, porém o mesmo prezou pela qualidade do sinal, que foi "bom", ou seja, valor 6 demonstrado no gráfico. A palavra/expressão confeccionada foi "jogar futebol".

O terceiro sinal gerado foi através da palavra "sexo", onde o usuário demonstrou grande evolução, tanto no tempo quanto na qualidade, pois gastou somente 15 minutos e a qualidade do sinal foi ótima, ou seja, valor 8 do gráfico.

No último sinal, foi soletrado o nome do usuário, ou seja, foi confeccionada a palavra "Jorge". Neste teste o resultado foi surpreendente, pois o usuário demonstrou grande familiaridade com a ferramenta, tendo como valores: tempo: 12 minutos e qualidade: 10.

Ao final dos testes, pode-se perceber que o usuário leigo, assim como a professora de LIBRAS, cresceu em escala ascendente em termo de qualidade, mostrado também que o tempo gasto está intimamente ligado a dificuldade/facilidade do sinal, e qualidade esperada.

\subsection{Com o surdo:}

Após a demonstração dos sinais através das orações traduzidas, Marcio Gleison Rodrigues dos Santos deu sua avaliação respondendo a duas questões: Qual foi o nível de compreensão das frases? Entre as opções: a) ruim; b) regular; c) bom; d) ótimo e e) excelente, representadas no gráfico como notas: a) 2; b) 4; c) 6; d) 8 e e) 10 . O mesmo respondeu a alternativa "c)", já que o usuário não domina completamente a língua de sinais, não conhecendo todas as palavras apresentadas no tradutor.

A segunda questão foi referente a seu nível de satisfação na utilização do tradutor, onde numa escala de 0 a 10, o mesmo assegurou satisfação total, ou seja, nota 10, devido à facilidade de manuseio da ferramenta e compreensão do que era proposto, e que viu nela um facilitador no processo de comunicação. 


\section{Gráfico 3: Avaliação do surdo}

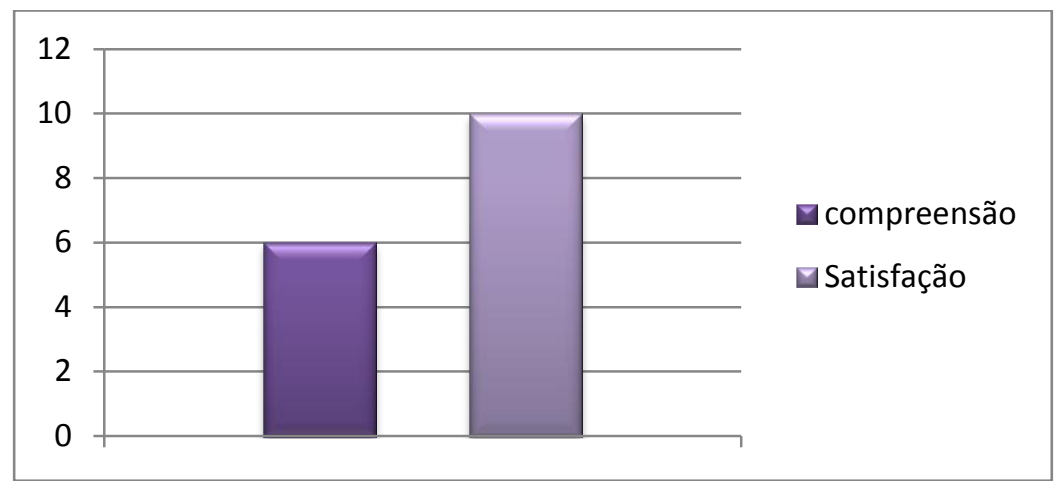

Desta forma, percebeu-se que os sinais produzidos no assistente tiveram uma boa aceitação por parte do usuário final, ou seja, o surdo Marcio Gleison Rodrigues dos Santos. Estes sinais foram testados através do tradutor FALIBRAS, mas podem ser incorporados a quaisquer tradutores. Foi de suma importância esta fase de avaliação do projeto, pois percebemos a necessidade de criar novas tecnologias para promover ações que saem do meio acadêmico e intervêm em problemas sociais.

\section{Considerações Finais}

A produção do AssistLibras foi realizada a partir da avaliação de uma solução para a problemática apresentada, depois de uma série de análises, percebeu-se que não havia na literatura e no mercado, alguma ferramenta que facilitasse a construção de sinais da LIBRAS. Para tal processo adotou-se o software mais adequado, tendo como apoio dois animadores. O assistente foi preparado para outro tipo de usuário, aquele que não tem experiência nem com animação gráfica nem com a língua de sinais, sendo sua parte mais importante a interface proposta, onde a mesma foi trabalhada através de análise de usabilidade e uma arquitetura que a proporcionasse.

Os experimentos realizados com os três usuários: professora de LIBRAS, usuário leigo e o surdo, tiveram resultados satisfatórios, pois desde a construção dos sinais até a visualização por parte do usuário final, o surdo, o AssistLibras mostrou grande aceitação e potencial de uso.

Apesar da hipótese lançada não ter sido confirmada em sua totalidade, por ser um problema de grande dimensão, e os resultados ainda restritos, espera-se que com a utilização de uma tecnologia assistiva adequada à realidade do surdo, seja possível promover sua comunicação com o meio social, possibilitando o advento da acessibilidade.

Assim sendo espera-se que este trabalho possa contribuir significantemente no meio acadêmico, como fonte de pesquisa a trabalhos futuros, mas principalmente no meio social, onde os sinais produzidos no assistente sejam utilizados em um tradutor, dentro da realidade do surdo brasileiro, promovendo sua acessibilidade à comunicação em seu meio social. 


\section{Referências}

ASSESSIBILIDADE BRASIL. Projeto de Computação Gráfica. Disponível em: <http://www.acessobrasil.org.br/index.php?itemid=198>. Acesso em mar/2012.

AUTODESK. Software de modelagem, animação e renderização em 3D. Disponível em <http://www.autodesk.com.br/adsk/servlet/pc/index?id=14644213\&siteID=1003425 $>$. Acesso em maio/2012.

BRASIL. 23,9\% da população tinha pelo menos um dos tipos de deficiência investigados. Censo IBGE 2010. Disponível em: http://www.ibge.gov.br/home/presidencia/noticias/noticia_visualiza.php?id_noticia= 2125\&id_pagina=1. Acesso em: maio/2012.

BRASIL. Casa Civil da Presidência da República. DECRETO No 5.626, DE 22 DE DEZEMBRO DE 2005. Capítulo II, Artigo 3․ Disponível em: http://www.planalto.gov.br/ccivil_03/_ato2004-2006/2005/decreto/d5626.htm. Acesso em: maio/2012.

BRASIL. Casa Civil da Presidência da República. LEI No 10.436, DE 24 DE ABRIL DE 2002. Disponível em: http://www.planalto.gov.br/ccivil_03/leis/2002/L10436.htm. Acesso em: maio/2012.

CAMELO, Dioclécio. Conceitos de Animação em Flash. CFSF - FUCAPI:: Design de Interface Digital:: Computação Gráfica 1. Amazonas, disponível em: <ftp://ftpaluno.umc.br/Redacao_Prof/Andruchack/.../Conceitos.pdf >, acesso em $\operatorname{mar} / 2012$.

CAPOVILLA, Fernando César; RAPHAEL, Walkiria Duarte. Dicionário Enciclopédico Ilustrado Trilíngue: Língua de Sinais Brasileira. 2. Ed. São Paulo: Edusp, 2001. p. 1632 .

EKMAN, P. Facial expressions. In: DALGLEISH, T.; POWER, T. (Ed.). The Handbook of Cognition and Emotion. Sussex, Reino Unido: John Wiley \& Sons Ltd: [s.n.], 1999. p.301-320.

FENEIS - Federação Nacional de Educação e Ingegração dos Surdos; WFD - World Federation of the Deaf (Federação Mundial dos Surdos). Disponível em: http://www.feneis.org.br/page/wfd.asp. Acesso em: jun/2012.

FRIAS, Elzabel Maria Alberton; MENEZES, Maria Christine Berdusco. Inclusão Escolar do Aluno com Necessidades Educacionais Especiais: contribuições ao professor do Ensino Regular. PDE, FAFIPA, 2008. Disponível em: http://www.diaadiaeducacao.pr.gov.br/portals/pde/arquivos/1462-8.pdf. jun/2012.

HAND TALK. Disponível em: http://www.handtalk.com.br/. Acesso em: ago/2012.

ICTS. Projeto Rybena. Disponível em: < http://www.grupoicts.com.br/ >. Acesso em $\operatorname{mar} / 2012$. 
ISO. Origens da Computação Gráfica, disponível em: <http://www.inf.pucrs.br/ pinho/CG/Aulas/Intro/intro.htm>, PUCRS, 2012, acesso em $\operatorname{mar} / 2012$.

JAQUES, P. A.; VICCARI, R. M. Estado da arte em ambientes inteligentes de aprendizagem que consideram a afetividade do aluno. Revista Informática na Educação: Teoria \& Prática, v. 8, n. 1, p. 15-38, 2005 a.

LIRA, Guilherme de Azambuja. Projeto TLIBRAS - Tradutor Português x LIBRAS (Língua Brasileira de Sinais). Acessibilidade Brasil. Disponível em: http://www.acessobrasil.org.br/index.php?itemid=39, acesso em ago/2012.

MANSSOUR, Isabel Harb; COHEN, Marcelo. Introdução à Computação Gráfica. RITA, Volume XIII, Número 2, 2006. P. 01. Disponível em: http://www.inf.pucrs.br/manssour/Publicacoes/TutorialSib2006.pdf. Acesso em: ago/2012.

PONTUAL, Jorge. Feira em Los Angeles reúne as novidades do mercado de games. Jornal da Globo. Disponível em: http://m.g1.globo.com/jornal-daglobo/noticia/2012/06/feira-em-los-angeles-reune-novidades-do-mercado-de-games. Acesso em: ago/2012.

PRODEAF. Soluções. Disponível em http://www.prodeaf.net/. Acesso em: mar/2012.

SÁ, Nídia Regina Limeira de. Cultura, poder e educação de surdos. 2. Ed. São Paulo: Paulinas, 2006.

SCHMIDT, K. L.; COHN, J. F. Human facial expressions as adaptations: Evolutionary questions in facial expression. American Journal of Physical Anthropology (Yearbook of Physical Anthropology), v. 44, n. S33, p. 3-24, 2001.

SCHNEIDER, Bruno de Oliveira; NEDEL, Luciana P. Simulação de Humanos Virtuais. Disponível em: http://www.inf.furb.br/gcg/projetos_pub/RAS/2RevisaoBibliografica.pdf. Acesso em ago/2012.

SILVA, Fernando Wagner da. Motion Capture - Introdução à Tecnologia. Laboratório de Computação Gráfica - LCG, COPPE/UFRJ - Engenharia de Sistemas e Computação. Disponível em: http://www.visgraf.impa.br/Projects/mcapture/publ/mctech/. Acesso em: mar/2012.

STUMPF, Marianne Rossi. Transcrições de Língua de Sinais Brasileira em signwriting. In: Letramento e minorias/ Organizadores: Lodi, Ana Cláudia B.

VALES, Lucila dos Santos. Pequeno Dicionário Regional de LIBRAS para Artes. Universidade Federal do Rio Grande do Sul. Faculdade de Educação. 2008. Disponível em: http://www.lume.ufrgs.br/bitstream/handle/10183/15665/000687612.pdf?sequence=1 . Acesso em: dez/12, às 22:00hs.

ZORZI, Ana Paula da Silva. A Comunicação entre Filhos Surdos e Pais Ouvintes. Livros Brasil, dez/2003. Disponível em: http://www.livrosbrasil.com.br/det_artigosoutros.asp?id_artigos=30 